\title{
Inhibition of complement activation by pexelizumab reduces death in patients undergoing combined aortic valve replacement and coronary artery bypass surgery
}

M. Carrier, MD, ${ }^{a}$ P. Ménasché, MD, ${ }^{m}$ J. H. Levy, MD, ${ }^{g}$ M. F. Newman, MD, ${ }^{f}$ K. M. Taylor, MD,

A. Haverich, $M D{ }^{c}{ }^{c}$ J. C. Chen, MD, ${ }^{d}$ S. K. Shernan, MD, ${ }^{e}$ F. Van de Werf, MD, ${ }^{h}$ M. van der Laan, MD, ${ }^{,}$T. G. Todaro, MD, ${ }^{,}$P. X. Adams, MD, ${ }^{k}$ and E. D. Verrier, MD'

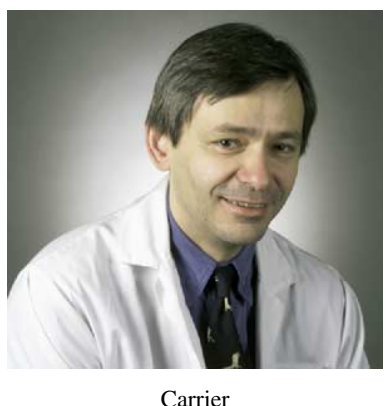

See related editorial on page 266 .

\begin{abstract}
From the Montreal Heart Institute, Montreal, Quebec, Canada; Hammersmith Hospital, NHLI Imperial College School of Medicine, London, United Kingdom; Hannover Medical School, Hannover, Germany; Kaiser Permanente Medical Center, University of Hawaii, Honolulu, Hawaii; Brigham and Women's Hospital, Harvard Medical School, Boston, Mass; Duke Clinical Research Institute, Duke University, Durham, NC; Emory University Hospital, Emory University, Atlanta, Ga; University Hospital Gasthuisberg, Leuven, Belgium; Proctor \& Gamble Pharmaceuticals, Egham, United Kingdom; Proctor \& Gamble Pharmaceuticals, Mason, Ohio; Alexion Pharmaceuticals, Inc, Cheshire, Conn; the University of Washington, School of Medicine, Seattle, Wash; and the Department of Cardiovascular Surgery, Hôpital Georges Pompidou, Paris, France.
\end{abstract}

Funding for the Pexelizumab for Reduction in Infarction and Mortality in Coronary Artery Bypass Graft surgery study was provided by the study's joint sponsors, Proctor and Gamble Pharmaceuticals, Cincinnati, Ohio, and Alexion Pharmaceuticals, Cheshire, Conn. Data for the current manuscript were provided by the sponsors to the authors at their request for their independent analysis and interpretation.

Received for publication June 14, 2005; revisions received Sept 23, 2005; accepted for publication Oct 7, 2005.

Accepted for publication Oct 7, 2005

Address for reprints: M. Carrier, MD, Research Center, Montreal Heart Institute, 5000 Belanger St East, Montreal, Quebec H1T 1C8, Canada (E-mail: michel.carrier@icmmhi.org).

J Thorac Cardiovasc Surg 2006;131:352-6

$0022-5223 / \$ 32.00$

Copyright () 2006 by The American Association for Thoracic Surgery

doi:10.1016/j.jtcvs.2005.10.011
Objective: We sought to evaluate the effects of pexelizumab, a C5 complement inhibitor, on death and myocardial infarction in patients undergoing combined aortic valve replacement and coronary artery bypass grafting surgery.

Methods: The Pexelizumab for Reduction in Myocardial Infarction and Mortality in Coronary Artery Bypass Graft surgery trial, a phase III prospective, randomized, double-blind, placebo-controlled study, enrolled 3099 patients at 205 centers. The primary end point was the composite of death, myocardial infarction, or both at postoperative day 30 in patients undergoing coronary artery bypass grafting without valve surgery. Postoperative myocardial infarction was defined as a creatine kinase MB fraction value of $100 \mathrm{ng} / \mathrm{mL}$ or greater, Q-wave myocardial infarction with a creatine kinase MB fraction value of $70 \mathrm{ng} / \mathrm{mL}$ or greater, or new Q-wave evidence of myocardial infarction by postoperative day 30 . Because patients undergoing coronary artery bypass grafting with a valve procedure were not included in the primary population, separate analysis of death and myocardial infarction was conducted in 218 patients undergoing combined aortic valve replacement and coronary artery bypass grafting surgery.

Results: Of the 353 patients randomized to any valve procedure, $106(61 \%)$ underwent combined aortic valve replacement and coronary artery bypass grafting in the pexelizumab treatment group compared with $112(63 \%)$ patients in the placebo group. Coronary artery bypass grafting was performed with 1 or more internal thoracic artery grafts in $139(64 \%)$ patients and with 1 or more saphenous vein grafts in $179(82 \%)$ patients. There were 4 (3.8\%) deaths in the pexelizumab group versus $11(9.9 \%)$ in the placebo group by postoperative day 30 and $6(5.7 \%)$ deaths in the active group versus $16(14.4 \%)$ in the placebo group by postoperative day $180(P=.107$ and $P=.043$, respectively, Fisher exact test). The incidence of myocardial infarction 30 days after surgical intervention was identical in the 2 groups, but the study was not designed to detect differences in this cohort of patients.

Conclusions: Inhibition of complement activation by pexelizumab resulted in a decreased mortality at 180 days among 218 patients who underwent combined aortic valve replacement and coronary artery bypass grafting surgery. Additional studies are warranted to confirm this decrease in mortality with pexelizumab in combined aortic valve replacement and coronary artery bypass grafting procedures.

$\mathrm{P}$ exelizumab is a novel recombinant, single-chain, and humanized anti-C5 monoclonal antibody fragment recently shown to decrease myocardial infarction (MI) and mortality rates after coronary artery bypass grafting (CABG). ${ }^{1}$ Experimental evidence suggests that inhibition of $\mathrm{C} 5$ conversion to $\mathrm{C} 5 \mathrm{a}$ 
and $\mathrm{C} 5 \mathrm{~b}$ can decrease the inflammatory reaction related to cardiopulmonary bypass $(\mathrm{CPB})$ and reperfusion of ischemic myocardium. ${ }^{2,3}$ Human studies have also shown a beneficial effect of C5 inhibition on inflammation markers, MI rate determined on the basis of creatine kinase MB fraction (CK-MB), and postoperative death in that setting. ${ }^{4}$

The Pexelizumab for Reduction in Infarction and Mortality in Coronary Artery Bypass Graft surgery (PRIMOCABG) trial enrolled 3099 patients, of whom 218 underwent combined aortic valve replacement (AVR) and $\mathrm{CABG}^{5}$ between January 2002 and February 2003. Patients undergoing combined valve and coronary artery surgery represent a complex subgroup of patients at a much higher risk for morbidity and mortality after surgical intervention compared with patients undergoing isolated CABG. The present study analyzes results of a subgroup of patients of the PRIMO-CABG trial. Patients who underwent combined $A V R$ and CABG and who were recruited in the PRIMOCABG trial formed the present group of interest for the study.

\section{Patients and Methods \\ Patient Population}

The study population, design, and main findings of the PRIMOCABG trial have already been published. ${ }^{5}$ In summary, the trial was conducted at 205 sites in North America and Western Europe. Eligible patients included those scheduled for CABG with or without concurrent valve surgery. The study included patients with one or more of the following baseline risk factors: urgent intervention, diabetes mellitus, female sex, prior CABG procedure, history of a neurologic event, history of congestive heart failure (New York Heart Association class III or IV), or history of 2 or more MIs (excluding patients who have had an MI within 48 hours of $\mathrm{CABG}$ ) or an MI that occurred 48 hours or more but 4 or fewer weeks before CABG. Exclusion criteria included planned aortic dissection repair, required aortic root reconstruction, or both; required salvage intervention; current cardiogenic shock; acute left ventricular, septal, or acute papillary muscle rupture; uncontrolled diabetes; history of renal failure and a serum creatinine value of greater than $3.0 \mathrm{mg} / \mathrm{dL}$; history of chronic hepatic failure, hepatic cirrhosis, or both; and a history of malignancy, known or suspected hereditary complement deficiency, and any active infection. The institutional review boards or equivalent approved the protocol at each site, and all patients provided written consent.

\section{Study Protocol}

Patients were randomized in a double-blind fashion to receive either intravenous pexelizumab $(2.0 \mathrm{mg} / \mathrm{kg}$ bolus followed by 0.05 $\mathrm{mg} \cdot \mathrm{kg}^{-1} \cdot \mathrm{h}^{-1}$ infusion for 24 hours) or placebo (placebo bolus followed by 24-hour infusion). Stratification occurred within each site and was based on whether valve surgery was planned, the type of valve surgery performed (mitral or other valve), and whether primary or repeat CABG was scheduled. Pexelizumab or placebo bolus was administered as soon as possible after anesthesia induction but no later than 10 minutes before CPB. Patients were followed for in-hospital adverse events and clinical end points. In

\begin{tabular}{|ll|}
\hline Abbreviations and & Acronyms \\
AVR & $=$ aortic valve replacement \\
CABG & $=$ coronary artery bypass grafting \\
CK-MB & $=$ creatine kinase MB fraction \\
CPB & $=$ cardiopulmonary bypass \\
MI & $=$ myocardial infarction \\
POD & $=$ postoperative day \\
PRIMO-CABG & $=$ Pexelizumab for Reduction in \\
& Infarction and Mortality in Coronary \\
& Artery Bypass Graft surgery \\
&
\end{tabular}

addition, patients were seen 14,30 , and 90 days after CABG surgery for adverse events and clinical outcomes and were contacted by telephone at 6 months to determine survival status.

\section{Study End Points}

For the present study focusing on patients undergoing AVR and CABG, death, MI, and complications were analyzed 30 and 90 days after surgical intervention, and survival status was also studied at 180 days. Death was defined as all-cause mortality. MI included both Q-wave and non-Q-wave MIs. A clinical events committee consisting of 3 expert cardiologists blinded to patient treatment assignment adjudicated all MIs. Through postoperative day (POD) 4, the diagnosis of a Q-wave MI required a new Q-wave persisting through POD 30 or associated with a peak CK-MB value of $70 \mathrm{ng} / \mathrm{mL}$ or greater; non-Q-wave MI required a peak CK-MB value of $100 \mathrm{ng} / \mathrm{mL}$ or greater within 96 hours postoperatively and without a new Q-wave. CK-MB measurements were collected at $4,8,12,16,24,72$, and 96 hours postoperatively and analyzed at a central core laboratory. Troponin I measurements were collected immediately before surgical intervention and on days 1, 2, and 4 after surgical intervention. Electrocardiograms were recorded at patient enrollment, as well as 48 and 96 hours and 14, 30, 90, and 180 days postoperatively. All electrocardiograms for the primary end point and prespecified secondary analyses were read at a central core laboratory. The pharmacodynamic effect of pexelizumab (inhibition of serum complement activity) was determined by using a standard total serum complement assay, as previously described. ${ }^{6}$

\section{Statistical Analyses}

The present report focused solely on patients recruited in the trial who underwent combined AVR and CABG. Patients who underwent isolated $\mathrm{CABG}$ and those who underwent $\mathrm{CABG}$ with another valve procedure at surgical intervention were excluded. The Wilcoxon rank sum test was performed on continuous data, and the Fisher exact test was used in comparing incidence rates. Survival analysis included Kaplan-Meier curve estimation.

\section{Results}

Patient Characteristics and Operative Data

Of the 218 patients undergoing AVR and CABG, 106 were randomized to receive intravenous administration of pexelizumab during the first 24 hours after surgical intervention 
TABLE 1. Patient characteristics

\begin{tabular}{lccr}
\hline & Pexelizumab (n= 106) & Placebo (n= 112) & $P$ value \\
\hline Age (y) & $73 \pm 8$ & $73 \pm 9$ & .635 \\
Female sex & $39(37 \%)$ & $43(38 \%)$ & .889 \\
NYHA class III and IV & $43(41 \%)$ & $53(48 \%)$ & .341 \\
Aortic valve insufficiency & $8(7.5 \%)$ & $12(10.7 \%)$ & .486 \\
Reoperative CABG & $15(14 \%)$ & $15(13 \%)$ & 1.000 \\
Prior myocardial infarction & $20(18.9 \%)$ & $27(24.1 \%)$ & .411 \\
\hline
\end{tabular}

NYHA, New York Heart Association; CABG, coronary artery bypass graft surgery.

TABLE 2. Operative data

\begin{tabular}{|c|c|c|c|}
\hline & Pexelizumab (n = 106) & Placebo (n = 112) & $P$ value \\
\hline Crossclamp time (min) & $106 \pm 3$ & $107 \pm 3$ & .979 \\
\hline CPB time (min) & $146 \pm 5$ & $147 \pm 4$ & .870 \\
\hline ITA grafts & $66(62 \%)$ & $73(65 \%)$ & .675 \\
\hline SV grafts & $92(87 \%)$ & $87(78 \%)$ & .111 \\
\hline No. of coronary artery grafts per patient & 2.4 & 2.2 & .336 \\
\hline
\end{tabular}

$C P B$, Cardiopulmonary bypass; ITA, internal thoracic artery; SV, saphenous vein.

TABLE 3. Adverse events and infection logs through 90 days

\begin{tabular}{|c|c|c|c|}
\hline & Pexelizumab $(n=104)$ & Placebo $(n=110)$ & $P$ value \\
\hline Atrial fibrillation & $28(26.9 \%)$ & $30(27.3 \%)$ & 1.000 \\
\hline Pleural effusion & $26(25 \%)$ & $26(23.6 \%)$ & .874 \\
\hline Acute renal failure & $4(3.8 \%)$ & $4(3.6 \%)$ & 1.000 \\
\hline Cardiac tamponade & $3(2.9 \%)$ & $2(1.8 \%)$ & .676 \\
\hline Complete AV block & $3(2.9 \%)$ & $1(0.9 \%)$ & .358 \\
\hline Septicemia & $2(2 \%)$ & $11(10.2 \%)$ & .019 \\
\hline Endocarditis & 0 & $1(0.9 \%)$ & 1.000 \\
\hline Pneumonia & $4(3.9 \%)$ & $13(11.9 \%)$ & .042 \\
\hline \multicolumn{4}{|l|}{ Sternal wound infection } \\
\hline Superficial & $2(2 \%)$ & $4(3.7 \%)$ & .684 \\
\hline Deep & $2(2 \%)$ & $1(0.9 \%)$ & .61 \\
\hline Wound infection at the leg vein harvesting site & $10(9.8 \%)$ & $5(4.6 \%)$ & .18 \\
\hline Urinary tract infection & $6(5.9 \%)$ & $17(15.7 \%)$ & .001 \\
\hline Stroke & $2(1.9 \%)$ & $9(8.2 \%)$ & .06 \\
\hline
\end{tabular}

$A V$, Atrial ventricular.

compared with 112 patients undergoing the same procedure who were allocated to the placebo group. Patients in both groups were mostly male and averaged 73 years old. Aortic stenosis was the main indication for AVR in the 2 groups of patients. Fourteen percent and $13 \%$ of the patients, respectively, underwent reoperative CABG (Table 1).

The average crossclamp and CPB times were similar in the 2 groups. The use of internal thoracic artery and saphenous vein grafts was also similar between the 2 groups (Table 2).

\section{Adverse Events and Pharmacodynamics}

The most common adverse events reported were atrial fibrillation and pleural effusion, with a similar incidence reported between groups (Table 3). Of interest, septicemia and pneumonia were more frequent in the placebo group compared with in the pexelizumab group. Endocarditis and sternal wound infection rates were similar in the 2 groups. Stroke rates were not statistically different between the 2 groups. Pexelizumab bolus and 24-hour infusion resulted in an inhibition of total serum complement hemolytic activity, which recovered to baseline levels 72 hours after surgical intervention (Figure 1).

\section{Clinical Outcomes}

Four patients (4/106 [3.8\%]) in the pexelizumab group and $11(11 / 112$ [9.9\%]) in the placebo group died within the first 30 days after surgical intervention $(P=.107)$, suggesting a $62 \%$, albeit not statistically significant, reduction in death 


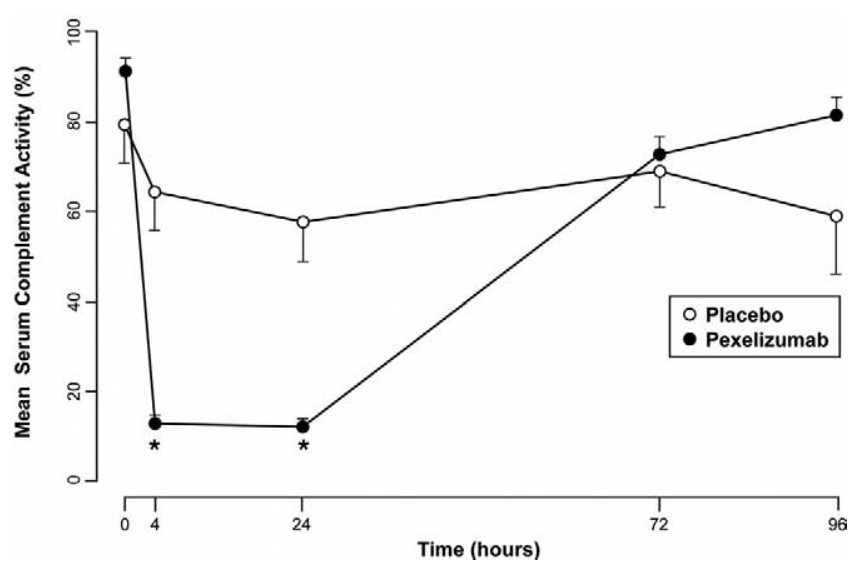

Figure 1. Serum complement activity measure with a standard complement hemolysis assay. ${ }^{*} \boldsymbol{P}<.01$ between the 2 groups at 4 and 24 hours, Wilcoxon rank sum test.

with pexelizumab. In the pexelizumab group $22(22 / 106$ [21\%]) patients and in the placebo group 23 (23/112 [21\%]) patients met the previously defined criteria for postoperative MI within the first 30 days after surgical intervention $(P=$ 1.000). Q-wave MI was documented in 7 (7/106 [6.6\%]) and $3(3 / 112[2.7 \%])$ patients, respectively $(P=.205)$. NonQ-wave MI was documented in $15(15 / 106$ [14.2\%]) and 20 $(20 / 112[17.9 \%])$ patients, respectively $(P=.64)$. The composite of death, MI, or both occurred in 23 patients (23/106 [22\%]) in the pexelizumab group and in 31 patients (31/111 [28\%]) in the placebo group $(P=.44)$

At 90-day follow-up, 5 patients $(5 / 106$ [4.7\%]) in the pexelizumab group and 15 patients $(15 / 112$ [13.5\%]) in the control group had died $(P=.034)$. Pexelizumab reduced the risk of death by $65 \%$. At 180 -day follow-up, mortality rates averaged $5.7 \%(6 / 106)$ and $14.4 \%(16 / 112)$, respectively $(P$ $=.043)$, a risk reduction of $60 \%$ among patients treated with pexelizumab (Figure 2).

The causes of death at 180-day follow-up among pexelizumab-treated patients were MI in 2 patients, renal failure in 1 patient, disseminated intravascular coagulation in 1 patient, multiorgan failure in 1 patient, and an unspecified cause in a last patient. Among the latter patients, one death from MI occurred at POD 31, and another occurred at POD 88 from renal failure. In the placebo group the causes of death at 180-day follow-up were cerebral complication (stroke and bleeding) in 3 patients, sepsis in 3 patients, respiratory failure in 2 patients, congestive heart failure in 2 patients, renal failure in 1 patient, cardiogenic shock in 1 patient, multiorgan failure in 1 patient, pulmonary artery trauma at surgical intervention in 1 patient, sudden cardiac death in 1 patient, and a noncardiac cause in a last patient. Among the latter patients, 1 patient died from cerebral complication at POD 32 after surgical intervention, 1 pa-
Aortic Valve Patient Survival Through POD 180

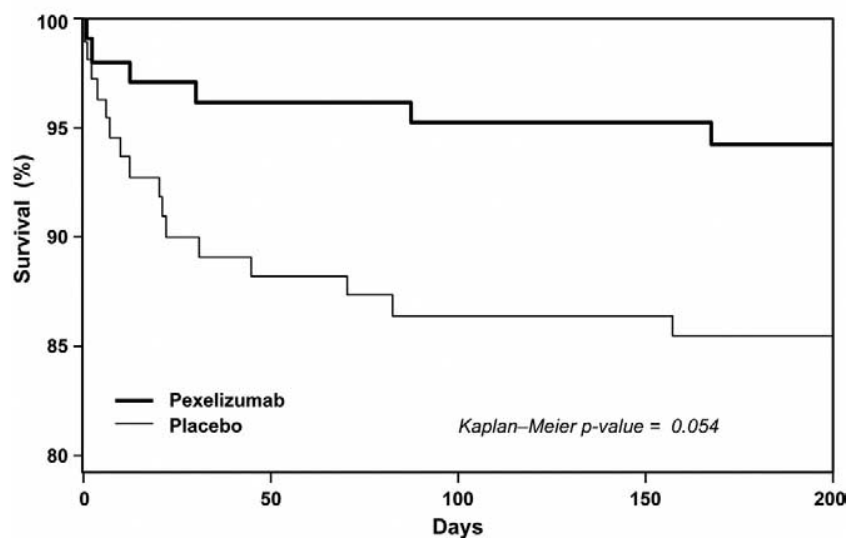

Figure 2. Actuarial survival.

tient died from sepsis at POD 46, 1 patient died from renal failure at POD 71, 1 patient died from cardiac arrest at POD 83 , and 1 patient died from a non-cardiac-related cause 158 days after surgical intervention.

\section{Discussion}

The present study showed that inhibition of complement activation by pexelizumab resulted in a decreased mortality at 90 and 180 days among patients who underwent combined AVR and CABG surgery. An important mechanism of cardiac injury during cardiac surgery is the excessive activation of the complement cascade related to ischemia and reperfusion, contact activation, and general systemic inflammation from CPB. Pexelizumab, a humanized anti-C5 complement antibody fragment, prevents cleavage of C5, thus mitigating the effects of the terminal complement components C5a and C5b-9 on vascular permeability, chemoattraction, and cellular activation, with subsequent cytokine release that might result in myocardial cell damage, systemic inflammation, and apoptosis. ${ }^{3}$

Fitch and colleagues ${ }^{4}$ first reported that sC5b-9, leukocyte activation through CD11b expression, myocardial injury determined on the basis of CK-MB measurements, and cognitive deficits determined with preoperative and postoperative tests were significantly reduced in patients who received pexelizumab. These authors have established that effective inhibition of human $\mathrm{C} 5$ has a profound antiinflammatory effect on patients undergoing $\mathrm{CABG}$ with CPB.

In a phase II prospective, multicenter, randomized, placebo-controlled trial, Shernan and associates ${ }^{1}$ suggested that MI and mortality could be decreased with pexelizumab in patients undergoing CABG. The PRIMO-CABG trial then enrolled 3099 patients: 2732 (88\%) underwent isolated CABG, and 353 (11\%) underwent combined valve surgery 
plus CABG, including 218 (7\%) undergoing AVR plus CABG. The primary end point of combined death or MI 30 days after surgical intervention was reduced by $18 \%$ in patients undergoing isolated CABG treated with pexelizumab. Pexelizumab was associated with a significant decrease in death or MI rate 180 days after surgical intervention. Although the study was not powered for detecting differences in mortality, death alone was not affected by pexelizumab in patients undergoing isolated $\mathrm{CABG}$.

Compared with patients undergoing isolated $\mathrm{CABG}$, our subgroup of patients undergoing AVR plus CABG was older, had a greater proportion of repeat $\mathrm{CABG}$, and was less likely to have experienced a preoperative MI. Of interest, mortality at 90 and 180 days after surgical intervention was decreased in patients treated with pexelizumab in our substudy patients who underwent combined AVR and CABG.

Several studies have reported difficulties in obtaining optimal myocardial protection during AVR because of associated left ventricular hypertrophy. ${ }^{7}$ Shernan and associates ${ }^{1}$ show that patients undergoing AVR plus CABG release more CK-MB after surgical intervention than patients undergoing isolated CABG (median CK-MB peak release of $50 \mathrm{ng} / \mathrm{mL}$ vs $30 \mathrm{ng} / \mathrm{mL}$, respectively). The greater release of CK-MB after AVR explains the high rate of MI reported in these patients compared with that seen in patients undergoing isolated CABG. The high rate of $\mathrm{MI}$ in the 2 groups (45/218 [21\%]), although mostly non-Q-wave, is related to the definition of postoperative MI based on the experience gained with the study of patients undergoing isolated CABG. Although Klatte and coworkers ${ }^{8}$ showed a relation between the release of CK-MB after isolated CABG and 6-month mortality in studying patients in the Guardian trial, whether this link is applicable to patients undergoing AVR remains to be proved.

The important limitation of the present study remains related to the subanalysis of the main trial. Therefore the difference in mortality in this report might not be clinically important, and a prospective randomized trial is required before cardiac surgeons adopt this therapy for this indication. The present post-hoc analysis raises the hypothesis that pexelizumab decreases early and 6-month mortality after combined AVR and CABG surgery. The effect of pexelizumab could be related to a decrease in the extent of myocardial ischemic injury and inhibition of inflammation cascades, leading to end-organ damage with the use of CPB in patients undergoing combined AVR and CABG. Further studies are needed to confirm this effect of the anti-C5 antibody pexelizumab.

\section{References}

1. Shernan SK, Fitch JC, Nussmeier NA, Chen JC, Rollins SA, Mojcik CF, et al. Impact of Pexelizumab, an anti-C5 complement antibody, on total mortality and adverse cardiovascular outcomes in cardiac surgical patients undergoing cardiopulmonary bypass. Ann Thorac Surg. 2004;77: 92-5.

2. Rinder CS, Rinder HM, Smith BR, Fitch JC, Smith MJ, Tracey JB, et al. Blockade of C5a and C5b-9 generation inhibits leukocyte and platelet activation during extracorporeal circulation. J Clin Invest. 1995; 96:1564-72

3. Vakeva AP, Agah A, Rollins SA, Matis LA, Li L, Stahl GL. Myocardial infarction and apoptosis after myocardial ischemia and reperfusion. Circulation. 1998;97:2259-67.

4. Fitch JC, Rollins S, Matis L, Alford B, Aranki S, Collard CD, et al. Pharmacology and biological efficacy of a recombinant, humanized, single-chain antibody C5 complement inhibitor in patients undergoing coronary artery bypass graft surgery with cardiopulmonary bypass. Circulation. 1999;100:2499-506.

5. Verrier ED, Shernan SK, Taylor KM, Van de Werf F, Newman MF, Chen JC, et al. Terminal complement blockade with pexelizumab during coronary artery bypass graft surgery requiring cardiopulmonary bypass. JAMA. 2004;291:2319-27.

6. Thomas TC, Rollins SA, Rother RP, Giannoni MA, Hartman SL, Elliott EA, et al. Inhibition of complement activity by humanized anti-C5 antibody and single-chain Fv. Mol Immunol. 1996;33:1389-401.

7. Jin XY, Gibson DG, Pepper JR. Early changes in regional and global left ventricular function after aortic valve replacement. Circulation. 1995;92(suppl II):II155-62.

8. Klatte K, Chaitman BR, Theroux P, Gavard JA, Stocke K, Boyce S, et al. Increased mortality after coronary artery bypass graft surgery is associated with increased levels of postoperative creatine kinasemyocardial band isoenzyme release. J Am Coll Cardiol. 2001; 38:1070-7. 